\title{
Coherence and dephasing in self-assembled quantum dots (invited)
}

Hvam, Jørn Märcher; Leosson, K.; Birkedal, Dan

Published in:

Third IEEE Conference on Nanotechnology, 2003. IEEE-NANO 2003.

Link to article, DOI:

10.1109/NANO.2003.1231730

Publication date:

2003

Document Version

Publisher's PDF, also known as Version of record

Link back to DTU Orbit

Citation (APA):

Hvam, J. M., Leosson, K., \& Birkedal, D. (2003). Coherence and dephasing in self-assembled quantum dots: (invited). In Third IEEE Conference on Nanotechnology, 2003. IEEE-NANO 2003. (Vol. 1, pp. 122-125). IEEE. https://doi.org/10.1109/NANO.2003.1231730

\section{General rights}

Copyright and moral rights for the publications made accessible in the public portal are retained by the authors and/or other copyright owners and it is a condition of accessing publications that users recognise and abide by the legal requirements associated with these rights.

- Users may download and print one copy of any publication from the public portal for the purpose of private study or research.

- You may not further distribute the material or use it for any profit-making activity or commercial gain

- You may freely distribute the URL identifying the publication in the public portal 


\title{
Coherence and Dephasing in Self-Assembled Quantum Dots
}

\author{
J.M. Hvam, K. Leosson and D. Birkedal \\ Research Center COM \\ Technical University of Denmark \\ DK-2800 Lyngby, Denmark \\ hvam@com.dtu.dk
}

\begin{abstract}
We measured dephasing times in InGaAlAs selfassembled quantum dots at low temperature using degenerate four-wave mixing. At $0 \mathrm{~K}$, the coherence time of the quantum dots is lifetime limited, whereas at finite temperatures pure dephasing by exciton-phonon interactions governs the quantum dot coherence. The inferred homogeneous line widths are significantly smaller than the line widths usually observed in the photoluminescence from single quantum dots indicating an additional inhomogeneous broadening mechanism in the latter.
\end{abstract}

Quantum dots; exciton coherence; dephasing; homogeneous and inhomogeneous line widths.

\section{INTRODUCTION}

With recent progress in the fabrication of self-assembled semiconductor quantum dots (SAQDs), the realization of a solid-state system with atom-like electronic and photonic properties has become feasible [1]. Along with the technological and scientific challenges associated with the fabrication and characterization of semiconductor quantum dots, their application potential has been widely forecast and discussed [2]. Many of the predicted properties of quantum dots (QDs) rely on a two-level (or few-level) picture of their electronic structure. The application of QDs in semiconductor optoelectronic devices like lasers and amplifiers has been triggered by predictions of lower thresholds, higher differential gain, less temperature dependence, lower chirp, higher modulation bandwidth etc. [3] In these applications, large ensembles of quantum dots are needed, and the relation between the homogeneous and the inhomogeneous broadening of the QD optical transitions plays an important role [4]. Another exciting application of the two-level nature of QDs would be as building blocks in quantum logic devices [5], where the coherence/dephasing time of the individual QDs is a crucial fundamental parameter. An equally important technological challenge is the control and positioning of the individual dots [6].

The dephasing time of QD transitions can be measured in frequency space as the homogeneous line width in e.g. photoluminescence (PL) spectra of single quantum dots [7,8]. Alternatively, it is measured in time domain by transient fourwave mixing (TFWM), or photon echo, experiments on an ensemble of quantum dots $[9,10]$. Both techniques have been pursued, and for weakly confined quantum dots formed by well-width fluctuations in narrow quantum wells this has led to consistent results for the low-temperature dephasing times of QD excitons [11,12]. For the more strongly confined excitons in SAQDs, however, four-wave mixing experiments have shown very long low-temperature dephasing times approaching 1ns $[9,10]$, whereas single-dot PL has shown line widths corresponding to dephasing times more than an order of magnitude shorter $[7,8]$.

In the present article we shall demonstrate that special care needs to be taken in order to ensure that the true homogeneous line width is measured in single-dot PL experiments consistent with the very long dephasing time measured in photon echo experiments [13]. One of the difficulties in performing nonlinear four-wave mixing experiments on quantum dot ensembles is the relatively small interaction volume normally available. This can be overcome by performing the QD TFWM in a waveguide geometry using heterodyne detection to separate the signal from the incident beams $[4,10]$ or by conventional two-beam TFWM on stacked multi-layer QDs [9]. In this work we shall concentrate on the latter.

\section{EXPERIMENTAL}

For the single-dot $\mathrm{PL}$ investigation, we used a single layer of MBE-grown $\mathrm{In}_{0.5} \mathrm{Al}_{0.04} \mathrm{Ga}_{0.46}$ As SAQDs embedded in $\mathrm{Al}_{0.08} \mathrm{Ga}_{0.92} \mathrm{As}$ barriers. Thin layers of AlAs were placed $20 \mathrm{~nm}$ above and $100 \mathrm{~nm}$ below the dot layers to prevent carrier diffusion to the surface and the GaAs substrate. In order to limit the detection area without disturbing the physical environment of the dots, a thin gold film with sub-micron sized apertures, fabricated by electron-beam lithography, was deposited on the surface of the sample. Another sample with ten layers of $\mathrm{In}_{0.5} \mathrm{Al}_{0.04} \mathrm{Ga}_{0.46} \mathrm{As}$ SAQD was grown for TFWM and time-resolved PL (TR-PL) experiments. Here an antireflection coating was applied to the sample back to prevent multiple reflections of the exciting beams.

PL was excited and detected through a microscope objective $(\mu \mathrm{PL})$ located inside a closed-cycle He cryostat. As excitation, we used two different continuous-wave light sources; a He-Ne laser $(632.8 \mathrm{~nm})$, and a frequency doubled $\mathrm{Nd}$ :YAG laser $(531 \mathrm{~nm})$. For high-resolution spectroscopy, the PL was dispersed in a 2-m Littrow spectrometer and detected 
with a cooled CCD camera. In TFWM and TR-PL experiments the sample was cooled in a liquid helium cryostat and excited with 120 -fs pulses from a Ti:sapphire laser. We used the $2 \mathbf{k}_{\mathbf{2}}-\mathbf{k}_{\mathbf{1}}$ transmission geometry and detected the signal using a silicon photo diode, with a low-noise preamplifier, and lock-in techniques. In TR-PL the sample was excited in the barriers and the PL was collected, dispersed, and synchronously detected using a streak camera with 2.5-ps time resolution.

\section{RESULTS}

\section{A. Luminescence}

The PL spectrum at $10 \mathrm{~K}$ from a $500-\mathrm{nm}$ aperture over a single layer of QDs is shown in the left inset of Fig. 1. The spectrum consists of a large number of well-resolved single lines. The section of the spectrum in the rectangular box is recorded at high spectral resolution and shown in Fig.1. The lines are well approximated with Lorentzians as shown with the solid line. The width (FWHM) is extracted for 54 lines and shown in the line-width histogram in the right inset of Fig. 1. Other apertures show similar line-width statistics. The most commonly measured line width is about $65 \mu \mathrm{eV}$. When corrected for the spectral resolution $(20 \mu \mathrm{eV})$, we obtain a typical dot line width of about $45 \mu \mathrm{eV}$, which is in good agreement with published data on line widths from selforganized quantum dots $[7,8]$.

In order to be able to correct for the temperature differences in single-dot and TFWM experiments, we have performed temperature dependent single-dot PL measurements. We find that the widths of the single-dot lines up to a temperature of approximately $40 \mathrm{~K}$ are well described by the linear relation

$$
\Gamma(T)=\Gamma(0)+a T
$$

where $a=0.5 \mu \mathrm{eV} / \mathrm{K}$ and $\Gamma(0)$ is described by a distribution peaking at $40 \mu \mathrm{eV}$.

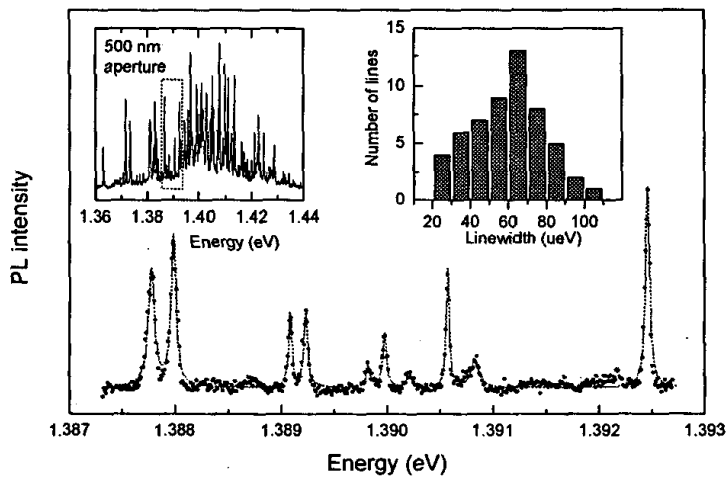

Figure 1. High-resolution PL spectrum at $10 \mathrm{~K}$ through a 500-nm aperture. The solid line is a Lorentzian fit to the data. The left inset shows all the lines from the 500-nm aperture. The right inset shows the line width statistics.

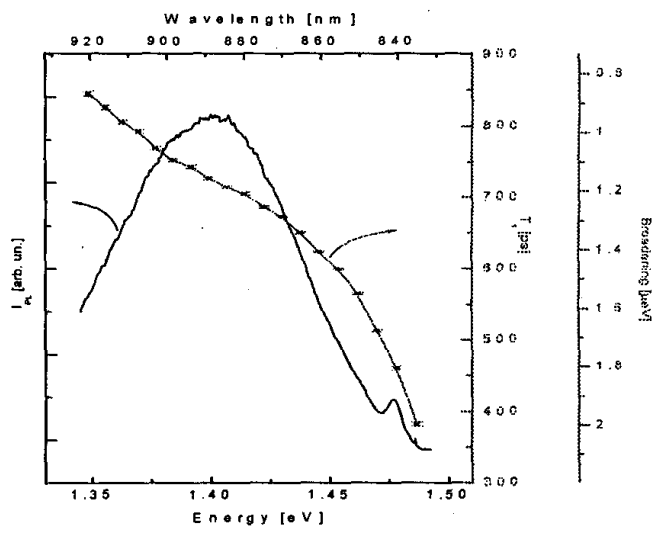

Figure 2. Time-resolved PL. The left axis shows the intensity of the timeintegrated $\mathrm{PL}$, and the right axes show the measured decay times and corresponding homogeneous broadenings at different spectral positions.

\section{B. Time-resolved luminescence}

In narrow quantum wells the homogeneous line width of localized excitons is dominated by lifetime broadening at the lowest temperatures. The question is whether the same holds true for SAQDs. We have performed time resolved PL spectroscopy on the ten-layer sample. This allows us to evaluate the PL decay time for individual spectral components across the entire luminescence band. We show in Fig. 2 the time-integrated PL spectrum together with the spectrally resolved decay times. The data show a spectral dependence of the PL decay times from $650 \mathrm{ps}$ at the high-energy side to $850 \mathrm{ps}$ at the low-energy side of the PL spectrum. The timeintegrated PL spectrum coincides with the PL obtained under continuous-wave excitation. The PL spectrum and PL decay times are nearly independent of excitation density over two orders of magnitude. We therefore conclude that the decay times observed are the lifetimes $T_{1}$ of the dot ground states. This allows us to calculate the contribution to the homogeneous line width from population decay to be about $1 \mu \mathrm{eV}$.

\section{Single-dot spectroscopy}

In the following we investigate the properties of the single dot spectra in more detail. The $\mu \mathrm{PL}$ spectra consist of a number of sharp lines, which are easily separated spectrally due to the large inhomogeneous broadening of the QD ensemble. A small part of such a spectrum is shown in the inset of Fig. 3 for three different excitation powers (531-nm excitation). At low power, we observe the ground-state transition lines $\left(\mathrm{X}_{1}, \mathrm{X}_{2}, \mathrm{X}_{3}\right)$, which increase linearly in intensity with the excitation power. Eventually, the intensities of these lines saturate and simultaneously additional lines appear (e.g. $\mathrm{XX}_{\mathrm{a}}, \mathrm{XX}_{\mathrm{b}}$ ) which increase quadratically with excitation power. We attribute these additional lines to biexciton states (not necessarily originating from the same dots as the exciton lines shown here). 


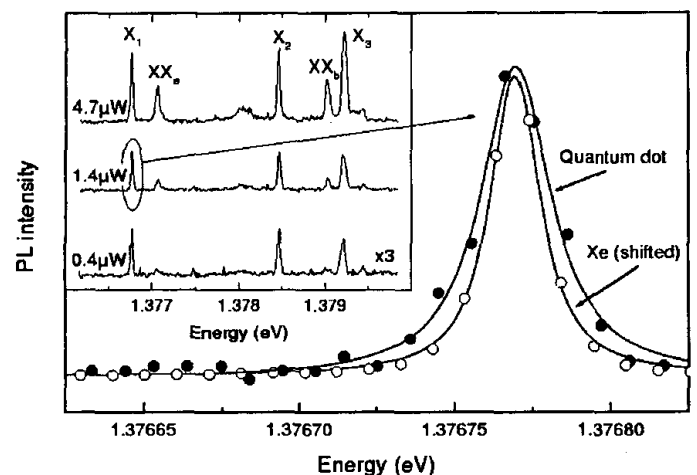

Figure 3. PL line from a single QD (filled circles) compared with the spectrometer response (open circles). Bothe lines are fitted by Lorentzian functions (solid lines). The inset shows spectra, recorded at differnet escitation powers. Exciton $(\mathrm{X})$ and biexciton $(\mathrm{XX})$ lines are indicated.

We focus our attention on the narrowest lines observed in the $\mu \mathrm{PL}$ spectra, e.g. the line $X_{1}$ shown in Fig. 3. We determine the spectrometer response function by recording a narrow line from a low-pressure Xe lamp. Both lines are well represented by Lorentzians with the widths of $23 \mu \mathrm{eV}$ and $17 \mu \mathrm{eV}$ (FWHM), respectively. Assuming that the measured PL line is a convolution of two Lorentzians, we obtain a deconvoluted line width of $6 \mu \mathrm{eV}$ for the QD emission. This value is in excellent agreement with the homogeneous broadening measured in the same type of dots at $10 \mathrm{~K}$ using TFWM [9], as we shall discuss in the next section. In similar TFWM experiments, Borri et al. found homogeneous line widths as low as $2 \mu \mathrm{eV}$ at $7 \mathrm{~K}$ in dots where the excitons are more strongly confined [10].

The TFWM experiments indicate that the dot ensemble is well characterized by a single dephasing time, suggesting that all dots have roughly the same homogeneous broadening. In the low-temperature $\mu \mathrm{PL}$ spectra, however, a distribution of line widths is found $[9,14]$, where only a fraction of the line widths are approaching the value measured in TFWM. For other transitions, such as $\mathrm{X}_{2}$ and $\mathrm{X}_{3}$ in Fig. 3, an additional broadening mechanism is therefore present. This may be spectral fluctuations, as have been observed in real time in III$\mathrm{V}$ [15] and II-VI [16] quantum dots and attributed to timevarying charge configurations in the vicinity of the dots. We observe minor variations $(<5 \mu \mathrm{eV})$ in the position of the PL lines in successively recorded spectra on a timescale of minutes, which is larger than the integration time used here $(10-30 \mathrm{~s})$, and therefore does not influence the line width. We note also that the broader lines typically have a Gaussian line shape, indicative of random fluctuations, in contrast to the narrower lines that have a Lorentzian shape. Thus, we conclude that dot-dependent spectral fluctuations, possibly due to charge fluctuations in the vicinity of the dots and occurring on a timescale faster than we can detect using our present set-up, are responsible for the observed distribution of line widths.

\section{Transient four-wave mixing}

The TFWM results are recorded at an excitation density of $190 \mathrm{~W} / \mathrm{cm}^{2}$, which is more than one order of magnitude larger than we usually apply to quantum well samples. This is due to the reduced absorbance of the QD layers, leaving only a small fraction $(<20 \%)$ of the quantum dots excited. The TFWM intensity still varies with excitation intensity to the third power in agreement with the underlying $\chi^{(3)}$ process.

The TFWM signal recorded at three different spectral positions of the laser (a, b, and c) and at $5 \mathrm{~K}$ is shown in Fig. 4. The inset shows the 5-K PL spectrum and the spectral positions of the laser for the three TFWM traces. A dominating signal at pulse overlap $(\tau=0)$ is common for the three TFWM traces. This signal is due to a non-resonant two-photon transition to the GaAs substrate and is unrelated to the quantum dots. For longer delays, a much weaker signal is observed. This weaker signal is seen to depend on the detuning of the laser with respect to the QD PL peak. For positive detuning (c) the TFWM signal is dominated by a fast component for short delays, and a much slower component for longer delays. The influence of the fast component is seen to diminish for more resonant tuning (b) and it disappears for negative detuning (a). The slow component, on the other hand, is observed to persist across the entire QD distribution.

The TFWM data are fitted well by a bi-exponential decay demonstrating that two distinct processes contribute to the TFWM signal. To obtain more quantitative information of the origin of the two processes we have recorded the TFWM signal for a number of different spectral positions of the laser. Each of these TFWM traces is fitted with a bi-exponential decay, and two decay times and two amplitudes are extracted.

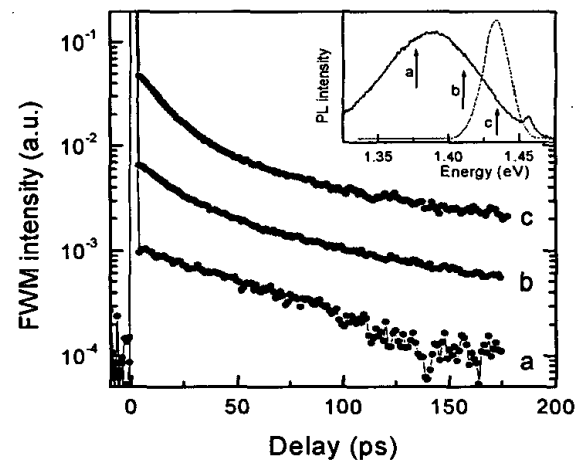

Figure 4. TFWM data for three different detunings of the laser $(a, b$, and $c)$ as indicated in the inset showing the PL spectrum and the laser spectrum centered at (c) 


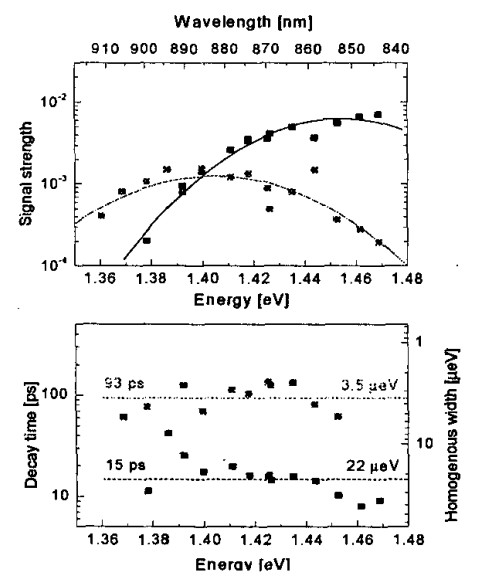

Figure 5. Decay-associated amplitudes (top) and decay times (bottom). The lines are Gaussian fits to the data points (top), and energy independent averages of the data points (bottom).

The results from the bi-exponential fits are shown in Fig. 5. The two decay-associated amplitudes are shown in the upper part. The amplitude of the slow component has a maximum at $1.405 \mathrm{eV}$, which nearly coincides with the peak of the low temperature PL spectrum (see inset of Fig. 4). In contrast, the amplitude of the fast component displays no clear maximum and merges into the onset of the wetting-layer transition. Since the low-temperature PL line-shape is in near agreement with the density of dot ground states (we assume that relaxation from excited states is fast and thermal redistribution of excitons between ground states is absent) we conclude that the slow component of the TFWM signal is due to excitation of dot ground states and the fast component is due to excitation of a mixture of wetting layer and excited states of the dots.

The decay times of the two contributions to the TFWM are plotted in the lower part of Fig. 5. The decay times of the fast and slow components are clearly separated in the plot. With the observed scatter in the data there is no basis for assuming any systematic spectral dependence of either of the decay times. Instead we calculate the average decay times, which are indicated with the horizontal lines. The average of the measured long decay times is 93ps. Since the TFWM signal is a time integrated photon echo this corresponds to a dephasing time $T_{2}=372 \mathrm{ps}$. This value is more than an order of magnitude larger than previously reported for SAQDs $[7,8]$ and excitons localized to potential fluctuations of narrow quantum wells $[11,12]$. The average $T_{2}$ can be converted to $\Gamma_{h}=3.5 \mu \mathrm{eV}$.

The short decay time has an average of $15 \mathrm{ps}$ or $\mathrm{T}_{2}=60 \mathrm{ps}$, which corresponds to a $\Gamma_{\mathrm{h}}=22 \mu \mathrm{eV}$. We speculate that the faster dephasing of the excited states is due to relaxation to the ground states. This channel is not available to the ground states, hence the difference of the two times. Assuming that relaxation processes dominate the homogeneous broadening of the excited states, this corresponds to an excited state lifetime of $30 \mathrm{ps}$, which is in agreement with our assumption of fast relaxation of the excited states.

\footnotetext{
Sponsored by the Danish Technical Science Research Council
}

\section{SUMMARY}

We have in TFWM experiments observed ultra-long coherence times of the dot ground state, which are more than one order of magnitude longer than found for localized excitons in narrow quantum wells. We confirm that dephasing times in excess of lns are attainable in self-assembled quantum dots, which should enable the demonstration of quantum logic operations in the solid state. We have also shown that a pure dephasing-limited line width can be observed in PL from III-V single quantum dots under proper excitation conditions.

\section{ACKNOWLEDGMENTS}

We would like to thank C.B. Sørensen and J.R. Jensen for sample growth and $\mathrm{K}$. Yvind for the anti reflection coatings.

\section{REFERENCES}

[1] D. Bimberg, M. Grundmann, and N.N. Ledentsov, Quantum Dot Heterostructures (John Wiley \& Sons, Chichester, 1999).

[2] D. Bimberg et al., "Novel infrared quantum dot lasers: Theory and reality," phys. stat sol. (b) 224 (2001) 787.

[3] J.M. Hvam, P. Borri, N.N. Ledentsov, and D. Bimberg, "Semiconductor quantum dot lasers and amplifiers," in Semiconductor lasers and optical amplifiers for lightwave communication systems," SPIE Proceedings Vol. 4871 (2002) 130.

[4] P. Borri, W. Langbein, J.M. Hvam, F. Heinrichsdorff, M.-H. Mao, and D. Bimberg, "Time-resolved four-wave mixing in InAs/InGaAs quantum-dot amplifiers under electrical injection," Appl. Phys. Lett. 76 (2000) 1380

[5] J.R. Guest et al., "Near-field coherent spectroscopy and microscopy of a quantum-dot system," Science 293 (2001) 2224.

[6] R. Notzel et al., "Uniform quantum-dot arrays formed by natural selffaceting on patterned substrates," Nature (London) 392 (1998) 56.

[7] K. Asaoka, Y. Ohno, S. Kishimoto, and T. Mizutani, "Microscopic photoluminescence study of InGaAs single quantum dots grown on (100) GaAs," Jpn. J. Appl. Phys. 38 (1999) 546.

[8] K. Ota, N. Usami, and Y. Shiraki, "Temperature dependence of microscopic photoluminecence spectra of quantum dots and quantum wells," Physica E 2 (1998) 573.

[9] D. Birkedal, K. Leosson, and J.M. Hvam, "Long lived coherence in selfassembled quantum dots," Phys. Rev. Lett. 87 (2001) 227401.

[10] P. Borri, W. Langbein, S. Schneider, U. Woggon, R.L. Sellin, D. Ouyang, and D. Bimberg, "Ultralong dephasing time in InGaAs quantum dots," Phys. Rev. Lett. 87 (2001) 157401.

[11] D. Gammon, E.S. Snow, B.V. Shanabrook, D.S. Katzer, and D. Park, "Fine structure splitting in the optical spectra of single GaAs quantum dots," Phys. Rev. Lett. 76 (2000) 3005.

[12] D. Gammon, E.S. Snow, B.V. Shanabrook, D.S. Katzer, and D. Park, "Homogeneous linesidth in the optical spectrum of single gallium arsenide quantum dots," Science 273 (1996) 87.

[13] K. Leosson, D. Birkedal, I. Magnusdottir, W. Langbein, and J.M. Hvam, "Homohenous linewidth of self-assembled III-V quantum dots observed in single-dot photoluminescence," Physica E 17 (2003) 1-6.

[14] K. Leosson, J.R. Jensen, J.M. Hvam, and W. Langbein, "Linewidth statistics of single InGaAs quantum dot photoluminescence," phys. stat. sol. (b) 221 (2000) 49.

[15] H.D. Robinson and B.B. Goldberg, "Light-induced spectral diffusion in single self-assembled quantum dots," Phys. Rev. B 61 (2000) 5086.

[16] R.G. Neuhauser, K.T. Shimizu, W.K. Woo, S.A. Empedocles, and M.G. Bawendi, "Correlation between fluorescence intermittency and spectral diffusion in single semiconductor quantum dots," Phys. Rev. Lett. 85 (2000) 3301 . 